\title{
Web-based Clinic Management System (CMS)
}

\author{
Jibrin Muhammad \\ Department of Mathematics and Computer Science \\ Sule Lamido University, Kafin Hausa. \\ Jigawa State, Nigeria
}

\author{
Salisu Garba \\ Department of Mathematics \& Computer Science \\ Sule Lamido University, Kafin Hausa. \\ Jigawa State, Nigeria
}

\begin{abstract}
In healthcare services, patient medical records are becoming large in size and the complexity of exchanging patient records such as prescription detail, referral data, diagnosis status and appointment schedules between various clinic-units can be a problem without a fully integrated system. Moreover, access to patient records requires privacy. Quite a number of works have been conducted on healthcare services to address issues such as medical record inconsistency, lack of immediate record storage and retrieval and paper-based approach. Consequently, this work tries to automate and enhance the clinical services of Sule Lamido University Clinic by developing a web-based application. The application will allow both the clinicians and patients to have access to electronic records easily, which will, in turn, minimize the cost, difficulties, and ineffectiveness of working with patients' records manually and to enhance the benefits and profits in running the clinic services. It can be used in decision making as it provides complete, reliable, accessible and understandable information pointing to the clinic progress and shortcomings. Initially, the problems of the current system were identified, the requirements were however specified and analyzed. Model of the system was designed and implemented using SDLC and finally, the system was tested and validated. The results revealed that the system complies with the specified requirements. Hence, the objectives under which the system is defined were achieved.
\end{abstract}

Keywords: CMS, Clinic, healthcare, HMS, management, System, information system, SLU

\section{INTRODUCTION}

Over the years, a wide range of literature has revealed the roles being played by Information System (IS) in healthcare services. [3] defines information systems based on two terms; these are information and system. In the process of advancing the idea, information is an analyzed data, while System is a collection of a finite set of elements joined together to' achieve objectives. More often than not, information systems normally consist of smaller systems known as subsystems, which all function towards ensuring the efficacy of the large systems [7].

Significant improvement in clinical workflow, administration as well as revenue enhancement are some among the benefits that outweigh the challenges of Information System in healthcare industries. Furthermore, another key thing to remember is access to clinical information; electronic access to medical records helps clinicians to save time and an enormous amount of resources. Thus, IS provides a complete, reliable, accessible and understandable information in a timely manner as well as in the effective management of patient medical records [4].

Consequently, the need for effective IS in healthcare services is considered substantial since it depicts organizational performance which in turn helps in predicting the future of possible challenges and at the same time provides appropriate solutions that can be built with streamlined operations towards the enhancement of proper administration and control over the untimely threats.

The CMS is an integration of patient information systems that captures and stores demographic, financial, and medical information from ancillary services such as registration, billing, lab, radiology, pathology, pharmacy, and transcription. The CMS also includes the network that links these systems, databases, interfaces, physician' order entry, electronic communication systems, and the clinical workstations [2].

Patient medical records accumulate during the clinical operations, information such as records of patients, treatment records, inventory stocks and data of other sources. As the complexity in healthcare services increases, healthcare practitioners find it difficult to manually exchange patient information from one file to another and from one healthcare practitioner to another. Hence, some among the major problems include; lack of immediate information storage and retrieval, poor preparation of accurate and prompt reports, lack of prompt updates, Error prone manual calculation.

In this paper, a web-based application for managing the services of Sule Lamido University Clinic is developed to deal with the above-mentioned problems. The rest of this paper is organized as follows; the related works are discussed in section 2. The proposed clinic management system is discussed in section 3 . The conclusion and future work are discussed in section 4 .

\section{RELATED WORK}

Tremendous efforts have been made to address problems in healthcare industries, and various researches have been embarked to provide solutions to the existing problems. They employed different approaches and tools for requirement analysis, project design and implementation as steps towards enhancing the effective processes needed for a modern healthcare system. As raised by [2], healthcare information systems are developed to meet the following goals: improve quality of care, reduce organizational expense, and produce' a data stream for electronic billing. The CMS meets these goals through workflow automation, connectivity, and consistent data sharing.

In research projects conducted by [8] and [9], there were no functionalities such as online consultation and report generation. However, the systems (mentioned above) need to be adjusted to comply with current requirements needed in modern healthcare services. In view of this, functionalities such as online consultation, appointment scheduling, diagnosis manager, report generator are added to the new system.

Hospitals and clinics can be regarded as organizations based on high technology and information' intensive processes. 
Such organizations are not hierarchically structured bureaucracies but are often based on democratic control mechanisms with institutionalized stakeholder influence in decision processes. It is also expected' that health care budgets and funding will depend significantly on the sophisticated patient and diagnosis classifications. The use of IT in diagnostic and treatment' processes will add to the development of networks of clinical, hospital and healthcare processes.

A considerable amount of literature has indicated that; healthcare management is a growing profession with increasing opportunities in both direct and non-direct care settings. [9] similarly opined that direct-care settings are those organizations that provide care directly to' a patient, resident or client who seeks' services from the organization. Nondirect care settings are not directly involved in providing' care to persons needing health services, but rather support the care of individuals through products and services made available to direct care settings.

Sule Lamido University clinic is currently operating manually. Its various services include; patient consultation, resuscitation and admission for observation during working hours, follow-up for hypertensive patients, treatment of minor trauma and counseling with medical issues, patient treatment services such as wound dressing and bandaging. The Clinic has full stocked pharmacy for provision of drugs and is being overseen by Pharmacist. The administrative section handles data processing services. Such services are being managed by assistant data processing officer and clerical officer using file processing approach. Therefore, with the ill-equipped Information System, the stock cannot be managed properly. Most of these problems can only be addressed if a proper Information System is developed.

\section{THE WEB-BASED CLINIC MANAGEMENT SYSTEM}

CMS is designed for both patients and practitioners. It will allow patients to create an appointment, print appointment card, generate medical reports, search for health tips, view doctor's profile, send emergency reports and chat with doctors online. The system will, however, allow practitioners to confirm, edit or cancel patient appointment request, diagnose patients, prescribe medicines to the patient, manage inventory, manage both patient and clinic staff records. As a step toward integrating the clinic operations, the proposed system composes of various modules, which are dependent on one another. The data flow between subsystems is illustrated in Figure 1. The sequence diagram of CMS appointment module is shown in Figure 2 and Use case diagram of CMS is shown in Figure 3.

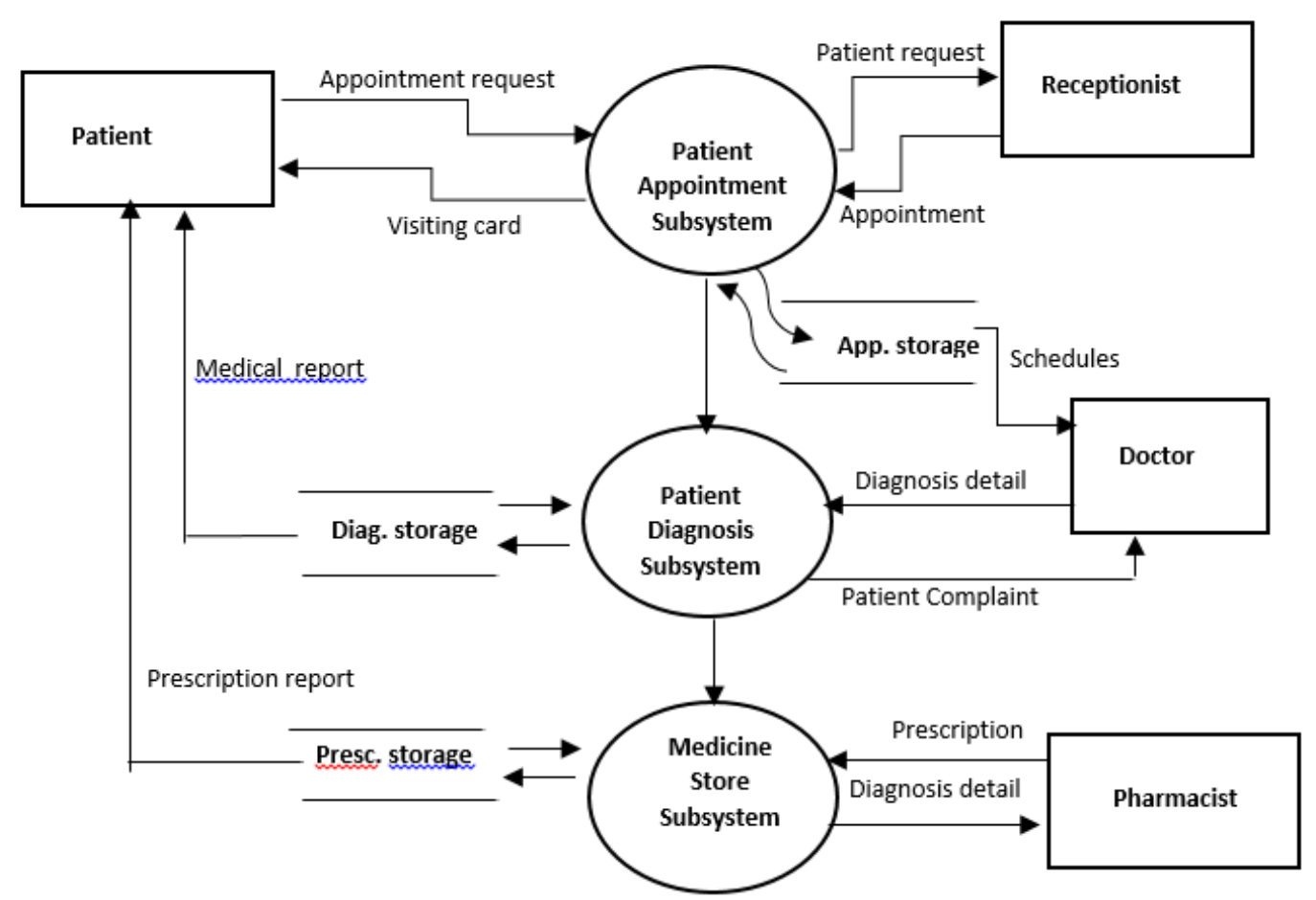

Figure 1: Level one DFD of CMS 
International Journal of Science and Engineering Applications

Volume 8-Issue 05,131-135, 2019, ISSN:-2319-7560

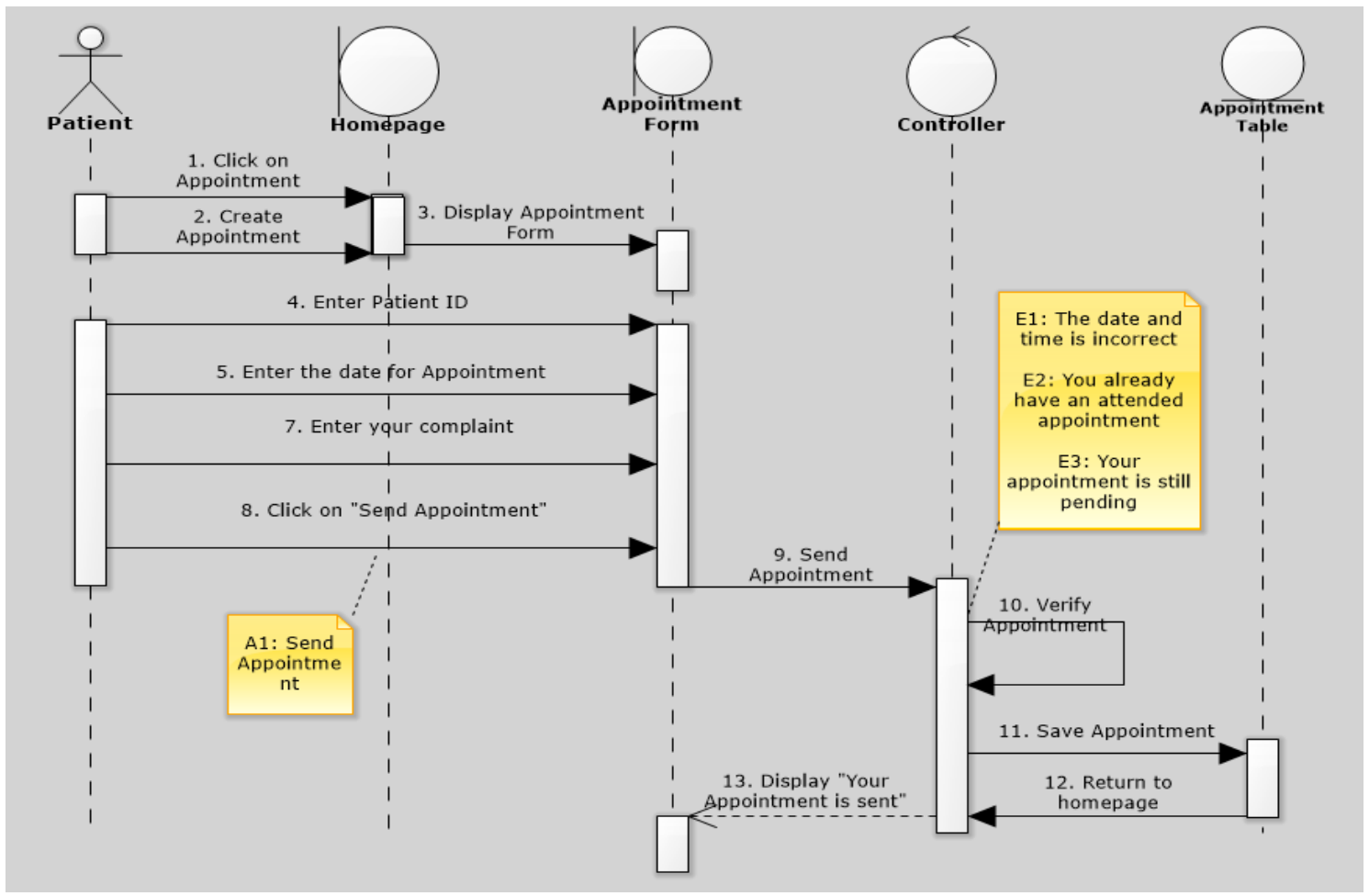

Figure 2: Sequence diagram of CMS Appointment Module

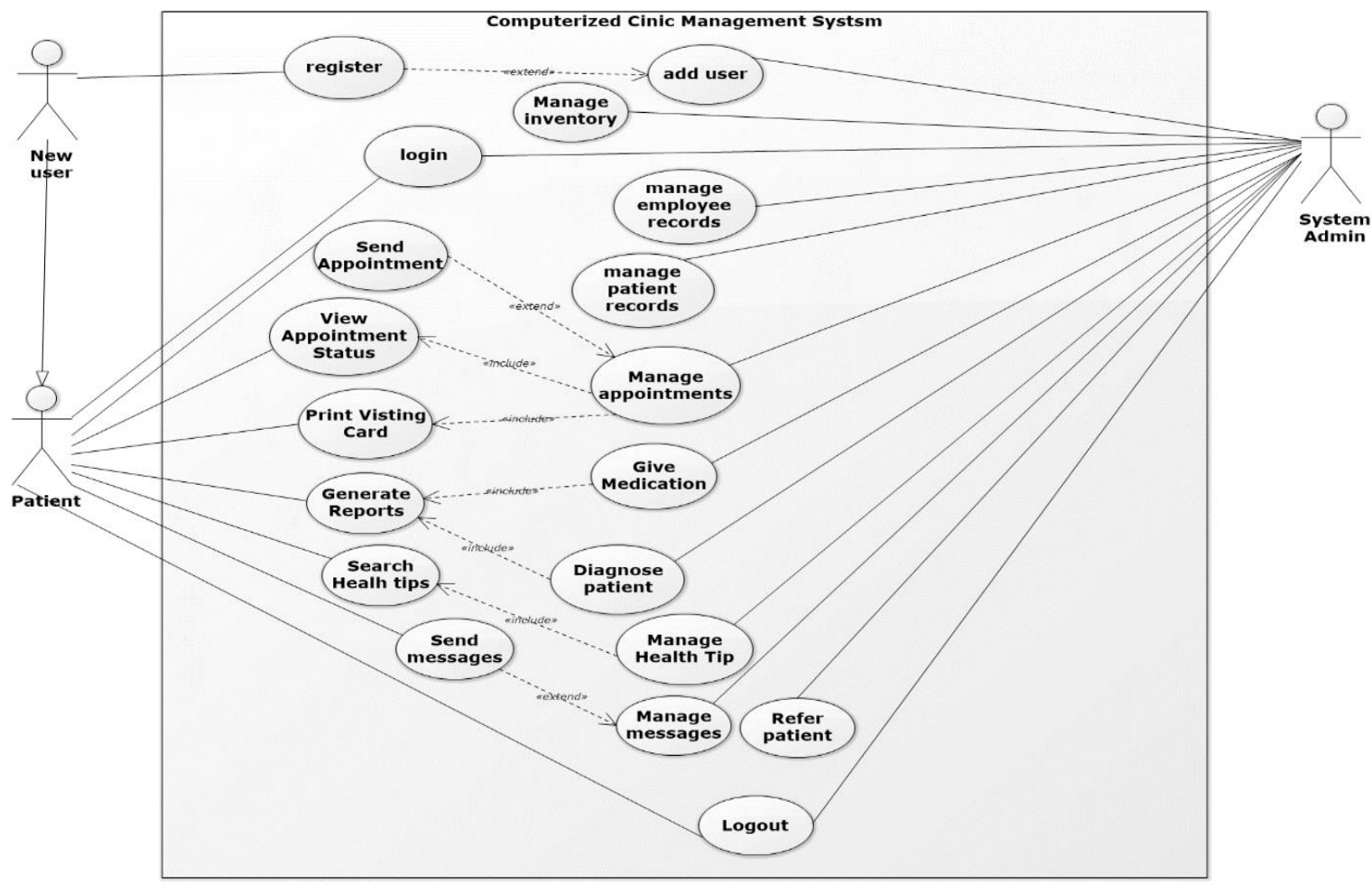

Figure 3: Use case diagram of CMS 
Table 1. The programming languages used in the system implementation

\begin{tabular}{|l|l|l|}
\hline Programming Language & Purpose & Environment for testing \\
\hline Hypertext Pre-Processor (PHP) & Servers-side scripting & Web browser and XAMPP server \\
\hline JavaScript & GUI behaviors and alerts & Web browser \\
\hline AJAX & HTTP requests & Web browser and XAMPP server \\
\hline Hypertext Markup Language (HTML) & Page structure & Web browser \\
\hline Cascading Style Sheet (CSC) & Presentation and styling & Web browser \\
\hline Structured Query Language (SQL) & Database queries & Web browser and MySQL DBMS \\
\hline
\end{tabular}

The database of this application is implemented using MySQL. It is an incredibly popular and powerful Relational Database Management System (RDBMS) that allows the creation of. MySQL is an open' source relational database management system (RDBMS) that uses Structured Query Language (SQL), the most popular language for adding, accessing, and processing' data in a database. MySQL is a true multi-user, multi-threaded SQL database server [12].

PHP is one of the programming language used in designing the program. Hypertext Pre-Processor (PHP) is a server-side scripting language, usually used to create web applications in combination' with a web server, such as Apache. PHP can also be used to' create command-line scripts akin to Perl or shell scripts, but such use is much less common than PHP's use as a web language [12]. The reason why PHP is used, because PHP is free, as there is' no 'cost to develop in and run programs made with PHP. PHP is compatible with the leading web servers: Apache HTTP Server for Linux/Unix and Windows' and Microsoft Internet Information Server. It also works with several' lesser-known servers. Specific web server compatibility with MySQL is not required since PHP will handle all the dirty work. There are other programming languages used such as JavaScript, SQL, HTML. The remaining details can be seen in Table 1 . The modules that make up the system are discussed in the subsections below.

\subsection{Appointment Scheduling Module}

This module is designed to have two sections (patient section and staff section), each of these sections is only accessible to the authorized user; patient accesses patient section and staff accesses staff section. The patient section allows the patient to send an appointment request, while the other section of the staff provides the detail of each patient request, the functionalities of confirming, editing and deleting the patient appointment request. If a patient appointment is confirmed, then he can print his visiting card instantly.

\subsection{Observation and Diagnosis Module}

After the appointment is confirmed, then the patient record goes to the observation module where he will be diagnosed by a doctor. However, this module saves the diagnosis record. After the diagnosis, the patient record may be sent to either admission module for the patient's admission, pharmacy module for medication or referral module if the patient is to be referred to a different hospital. Observation module is only accessible by staff.

\subsection{Patient Referral Module}

This module deals with the information sent from the observation module for patient referral. It is designed purposefully if the patient is needed to be referred to another clinic or hospital. Here in this module, other information like hospital facility to be referred and the result of the diagnosis is provided.

\subsection{Pharmacy and Inventory Module}

This acts as a medicine store, where medicines are kept. Patient from observation units is sent to the pharmacy for collecting medicines. There is a waiting panel for patients to wait while another patient is being attended. The module keeps track of the pharmacist who offers the medicine, the patient who collects the medicine, date and time for the medication. The module also includes a sub-module for prescribing patient during medication. It has a panel for adding and updating medicines if in case there is a need for any of such operations.

\subsection{Online Consultation Module}

This module acts as a messaging system where both patient and doctor discuss health-related issues in real time. It, however, provides a panel which shows active users online to indicate whether the user is available or not.

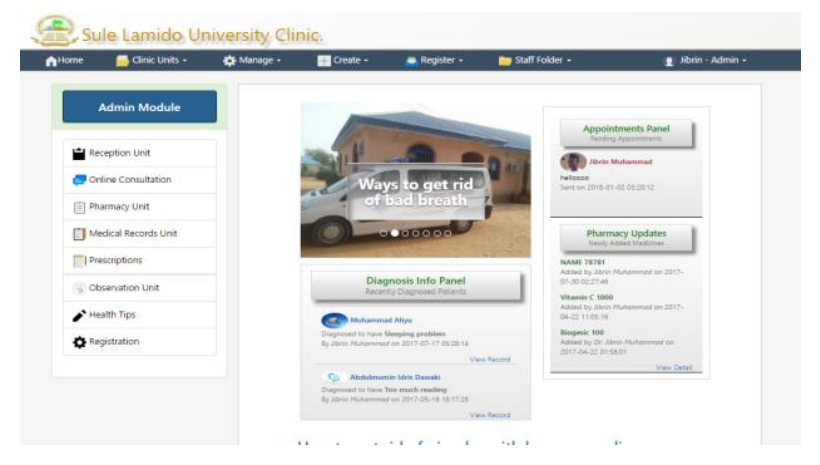

Figure 4: System Interface

\section{CONCLUSION}

CMS is designed and developed to automate the manual system of Sule Lamido University Clinic and oust the manual method of record/file keeping. Both the practitioners and patients will find this system useful, to aid in their computerization. Thus, the system will promote effective, efficient and quality services. Clinic Management System (CMS) currently focuses only on the relationships between patients, receptionist, physicians, and pharmacists. It is part of the future plan for this project to incorporate other healthcare facilities and professionals, such as laboratory technicians who perform, report tests and analysis requested by physicians. However, another plan is to enhance the system to investigate whether our Clinic and Pharmacy modules can be interfaced to applications supplied by pharmaceutical companies that provide information on medications and dosages. In addition to that, the future plan extends to develop a mobile phone application that will work with the system over the internet network. 


\section{ACKNOWLEDGMENTS}

Our thanks to the Sule Lamido University Clinic for and other staffs of the university who have contributed towards the development of this system.

\section{REFERENCES}

[1] Almasri R. \& Navathe B. S. (2010). Fundamentals of database systems sixth edition. Addison-Wesley, USA. ISBN-13:978-0-136-086208

[2] Erstad L. (2007). Analyzing Computer-based patient record: A review of literature; Journal of Healthcare Information Management Vol. 17 No. 4

[3] Kumar, P. K. (2006). Information System-Decision Making IndianMBA. Retrieved from http://www.indianmba.com/Faculty_Column/FC307/fc30 7.html on April 2, 2017

[4] Besson, P., Rowe, F. (2012). Strategizing information systems-enabled organizational transformation: A trans disciplinary review and new directions. Journal of Strategic Information Systems, 21, 103-124

[5] Hosseini, J. (2005). Strategic Technology Planning for the e-Commerce Enabled Enterprise. International Conference on Information Technology: Coding and Computing (ITCC'05).

[6] Jahangir, K. (2005). Improving organizational best practice with information systems. Knowledge
Management Review. Retrieved from http://findarticles.com/p/articles/mi_qa5362/is_200501/ai _ n21371132/ on March 4th, 2017

[7] Babu, K.V.S.N.J., \& Sekhar, B.M.R (2012). MIS. Vs. DSS in Decision Making, Global Journal of Management and Business Research, Volume 12.

[8] Basir, A. A. B. (2015). Darul Ridzuan Clinic Management System (Doctoral dissertation, Universiti Malaysia Sabah).

[9] Lau, F., Price, M., \& Keshavjee, K. (2011). Making sense of health information system success in Canada. Healthc Q, 14(1), 39-46.

[10] Lingham, L. (2006). Managing a business/ Management information system. All Experts. Retrieved from http://en.allexperts.com/q/ManagingBusiness1088/manag ement-information-system.htm on March 4th, 2017

[11] O’ Brien, J.A.,\& Marakas, G.M. (2007). Management information systems-10th edition. McGraw-Hill/Irwin, The McGraw-Hill Companies.

[12] Suehring S. (2009). PHP6 and MySQL6 bible. Wiley Publishing, Inc., Indianapolis, Indiana ISBN: 978-0-47038450-3

[13] S.K, J. (2009). System analysis and design. New Delhi, India: S.K \& Sons. 